\title{
Dynamic competition between island growth and coalescence in metal-on-insulator deposition
}

\author{
Bo Lü, Viktor Elofsson, Peter Münger and Kostas Sarakinos
}

\section{Linköping University Post Print}

\section{Tweet}

N.B.: When citing this work, cite the original article.

Original Publication:

Bo Lü, Viktor Elofsson, Peter Münger and Kostas Sarakinos, Dynamic competition between island growth and coalescence in metal-on-insulator deposition, 2014, Applied Physics Letters, (105), 16, 163107-1-163107-5.

http://dx.doi.org/10.1063/1.4900575

Copyright: American Institute of Physics (AIP) http://www.aip.org/

Postprint available at: Linköping University Electronic Press

http://urn.kb.se/resolve?urn=urn:nbn:se:liu:diva-112133 


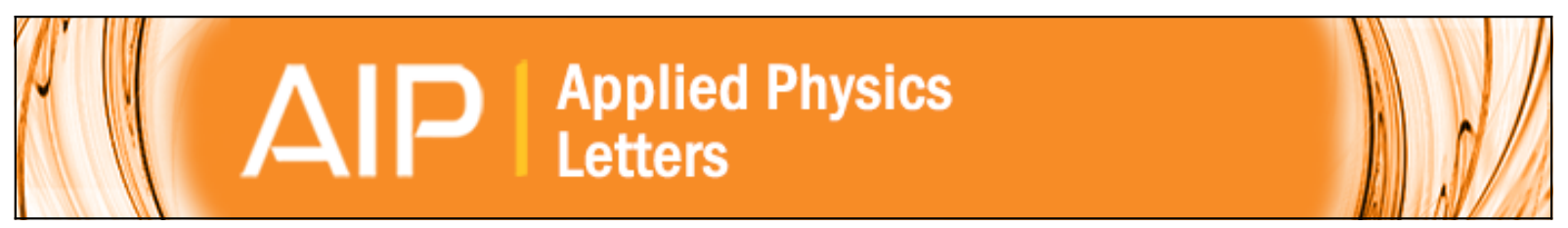

\section{Dynamic competition between island growth and coalescence in metal-on-insulator} deposition

B. Lü, V. Elofsson, E. P. Münger, and K. Sarakinos

Citation: Applied Physics Letters 105, 163107 (2014); doi: 10.1063/1.4900575

View online: http://dx.doi.org/10.1063/1.4900575

View Table of Contents: http://scitation.aip.org/content/aip/journal/apl/105/16?ver=pdfcov

Published by the AIP Publishing

\section{Articles you may be interested in}

Nanometer-scale molecular deposition using the surface Plasmon polariton interference field on a metal film Appl. Phys. Lett. 98, 093105 (2011); 10.1063/1.3560306

Atomic force microscopy study of the growth mechanisms of nanostructured sputtered Au film on $\mathrm{Si}(111)$ :

Evolution with film thickness and annealing time

J. Appl. Phys. 107, 104321 (2010); 10.1063/1.3428467

Temperature-induced chaos during nanorod growth by physical vapor deposition

J. Appl. Phys. 105, 094318 (2009); 10.1063/1.3116720

Quantitative determination of tensile stress creation during island coalescence using selective-area growth J. Appl. Phys. 97, 083530 (2005); 10.1063/1.1870109

Growth and characterization of nanoscale $3 \mathrm{C}-\mathrm{SiC}$ islands on Si substrates

J. Appl. Phys. 85, 3565 (1999); 10.1063/1.369772

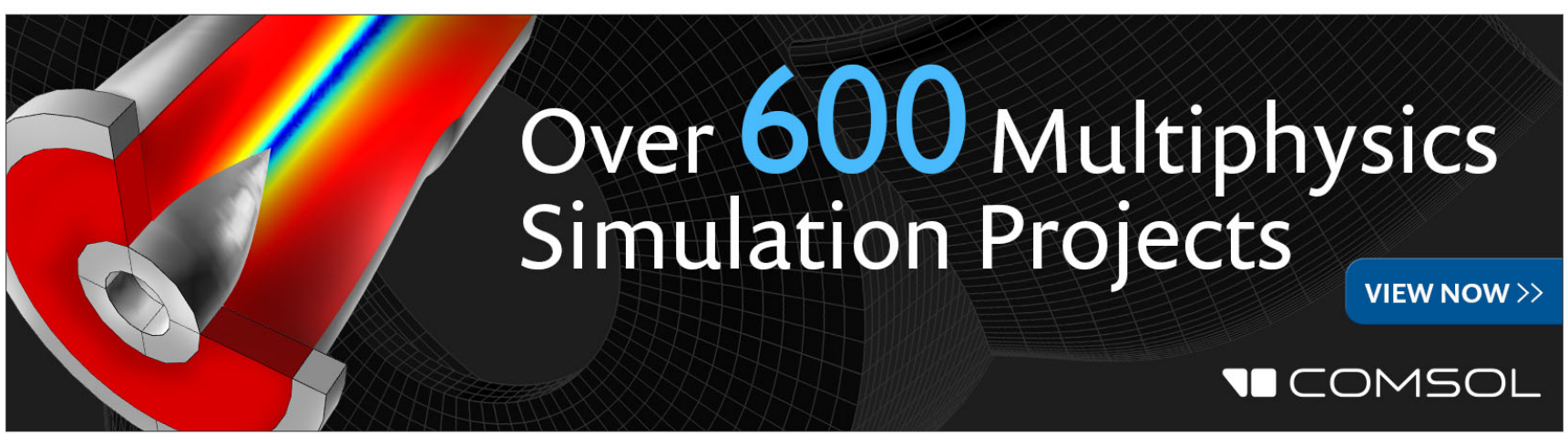




\title{
Dynamic competition between island growth and coalescence in metal-on-insulator deposition
}

\author{
B. Lü, V. Elofsson, E. P. Münger, and K. Sarakinos \\ Department of Physics, Chemistry and Biology, Linköping University, SE 581 83, Linköping, Sweden
}

(Received 15 September 2014; accepted 14 October 2014; published online 23 October 2014)

\begin{abstract}
The morphology of thin metal films and nanostructures synthesized from the vapor phase on insulating substrates is strongly influenced by the coalescence of islands. Here, we derive analytically the quantitative criterion for coalescence suppression by combining atomistic nucleation theory and a classical model of coalescence. Growth simulations show that using this criterion, a coalescence-free growth regime can be reached in which morphological evolution is solely determined by island nucleation, growth, and impingement. Experimental validation for the ability to control the rate of coalescence using this criterion and navigate between different growth regimes is provided by in situ monitoring of $\mathrm{Ag}$ deposition on $\mathrm{SiO}_{2}$. Our findings pave the way for creating thin films and nanostructures that exhibit a wide range of morphologies and physical attributes in a knowledge-based manner. (c) 2014 AIP Publishing LLC.
\end{abstract}

[http://dx.doi.org/10.1063/1.4900575]

Growth of thin films and nanostructures from the vapor phase starts with nucleation of isolated atomic islands. Further vapor condensation causes island growth, which competes with nucleation, determining the saturation island density, and thus characteristic size and separation of objects on the growing surface. ${ }^{1}$ However, subsequent growth is primarily dictated by coalescence, which occurs when two or more neighboring islands impinge and merge into a single larger island. This process evidently results in decrease of the island density, erasing morphological features attained during the nucleation stage. Moreover, in the case of Volmer-Weber or 3D growth, typically encountered during condensation of metal vapor on an insulating substrate, coalescence entails a contraction of base area as the new island undergoes shape equilibration. This acts to delay consecutive impingements, and hence also film percolation and formation of a continuous film. ${ }^{2-5}$ The result is a larger film thickness upon complete substrate coverage and higher surface roughness, the latter because coalescence broadens the island size distribution. $^{6-8}$ Since the rate of coalescence is finite, depending on island size, material, and growth temperature, ${ }^{9-11}$ it is in direct competition with the intrinsic island growth rate. This is in turn is set by the deposition rate and adatom diffusivity. A quantitative description of the competition dynamics may allow the rate of coalescence during vapor condensation to be controlled. In this way, the microstructural evolution can be fine-tuned using both nucleation and coalescence to access a larger palette of morphologies and physical attributes.

The classical treatment of coalescence employs the theories of Nichols and Mullins, in which mass transport between cap-shaped islands is solely based on surface self-diffusion, and the "strength" of coalescence is quantified by 9

$$
B=D_{S} \gamma \Omega^{2} \rho / k_{B} T\left(\mathrm{~m}^{4} / \mathrm{s}\right),
$$

where $D_{s}$ is the (isotropic) surface self-diffusivity, $\gamma$ is the (isotropic) surface tension, $\Omega$ is the atomic volume, $\rho$ is the planar atomic density, $k_{B}$ is the Boltzmann constant, and $T$ is the temperature. The main benefit of this description lies in its simple quantification of the coalescence completion time between two impinging islands, as ${ }^{12}$

$$
\tau_{\text {coal }}=R^{4} / B(\mathrm{~s})
$$

where $R[\mathrm{~m}]$ is the radius of the smaller island in a coalescing pair. Taking the limit $B \rightarrow \infty$ reproduces the droplet growth kinetics described by Family and Meakin, ${ }^{13}$ which was recently reported for room temperature vapor phase deposition of aluminum on both $\mathrm{SiO}_{2}$ and sapphire substrates. ${ }^{14}$ As $R$ increases with time, while $B$ is constant, $\tau_{\text {coal }}$ eventually becomes so large that further coalescence events cannot be completed and percolation ensues. ${ }^{3-5}$ Previous studies have questioned the validity of Eq. (2) for growth temperatures below the film material's roughening transition temperature. ${ }^{11,15-17}$ This causes the formation of nanoscale faceted islands, where the assumption of isotropic surface selfdiffusion is invalid. However, this uncertainty does not invalidate the existence of competition between island growth and coalescence, and thus, Eq. (2) can be used as a good approximation to illustrate the dynamics of this competition.

To determine the growth dynamics, scaling behaviors of film thicknesses at characteristic growth transitions are commonly studied as functions of deposition conditions. ${ }^{3,18,19}$ The so called elongation transition, which occurs shortly before percolation sets in, signifies when the substrate surface is largely covered by isolated, elongated structures comprised of islands undergoing coalescence. The film thickness (in monolayers, $\mathrm{ML}=$ atoms per unit area) at this transition can be related to strength of coalescence and the deposition rate through ${ }^{3,4}$

$$
\theta_{\text {elong }} \propto(B / F)^{1 / 3}(\mathrm{ML}),
$$

where $F(\mathrm{ML} / \mathrm{s})$ denotes the vapor flux to the substrate (i.e., deposition rate). Equation (3) was derived by comparing the rate of islands merging due to coalescence (Eq. (2)) to the rate of islands impinging due to growth and is therefore an 
indication that coalescence can be suppressed if the island growth rate is sufficiently large. For some materials such as $\mathrm{Pd}$, coalescence may be an intrinsically slow process (in the present context, having a small $B$ value) such that no coalescence events are completed during growth. ${ }^{20,21}$ In such cases, previous studies based on growth simulations have shown a different scaling relation for $\theta_{\text {elong }}$ as ${ }^{18,22}$

$$
\theta_{\text {elong }} \propto(D / F)^{1 / 7}
$$

where $D$ is diffusivity of deposited adatoms on the substrate, given by

$$
D=\nu_{0} a^{2} \exp \left(-E_{D} / k_{B} T\right)\left(\mathrm{a}^{2} / \mathrm{s}\right),
$$

where $\nu_{0}$ is the attempt frequency (typically of the order of $\left.5 \times 10^{12} \mathrm{~Hz}\right), a$ the minimum translational distance on the substrate and $E_{D}$ the substrate diffusion barrier.

The scaling expressions in Eqs. (3) and (4) indicate that for a given material, the role of coalescence during growth can be controlled by tuning the process parameters $F, D$, and $B$. The first step towards achieving this is to identify a quantitative criterion, as a function of the aforementioned parameters, for crossing between growth regimes where coalescence is either active or suppressed (illustrated by the scaling expression in Eqs. (3) and (4), respectively). In the present work, we derive this criterion by combining the nucleation and coalescence theories outlined thus far to express the rates of island growth and coalescence on an atomistic level. The theoretical results are validated against scaling behaviors of transition film thicknesses at various growth conditions from kinetic Monte Carlo (KMC) simulations and experimental results from in situ monitoring of $\mathrm{Ag}$ vapor deposition on $\mathrm{SiO}_{2}$.

The competition between island growth and coalescence in the later stages of growth, where nucleation is negligible, can be described analytically by comparing the rate of atoms added to each island $F / N$, where $N$ is the island density, to the rate of atoms leaving an island in a coalescence process $S / \tau_{\text {coal }}$, where $S$ is the average island size in number of atoms. ${ }^{23}$ In this way, the coalescence-free regime is dominated by the former rate while the coalescence-controlled regime is dominated by the latter rate. Analytically, we assume these rates to be equal at an ideal crossover between these regimes

$$
F / N=S / \tau_{\text {coal }}
$$

The effects of growth and coalescence competition are most prominent at the elongation transition; thus, we are interested in finding values of $N, S$, and $\tau_{\text {coal }}$ at this thickness. By substituting $\tau_{\text {coal }}$ with Eq. (2) $\left(\tau_{\text {coal }}=R^{4} / B\right)$ using $R=(3 S \Omega / 2 \pi)^{1 / 3}$ and $S=\theta / N$, Eq. (6) at the elongation transition can be expressed as

$$
F_{\text {crit }}=(2 \pi / 3 \Omega)^{4 / 3} B N_{\text {elong }}^{4 / 3} / \theta_{\text {elong }}^{1 / 3},
$$

where $F_{\text {crit }}$ is the specific deposition rate at the crossover for given $N_{\text {elong }}, \theta_{\text {elong }}$, and $B$. Next, we express $\theta_{\text {elong }}$ quantitatively by using the relations $S=\theta / N$ and $R=(3 S \Omega / 2 \pi)^{1 / 3}$ once more to find

$$
\theta_{\text {elong }}=N_{\text {elong }} 2 \pi R_{\text {elong }}^{3} / 3 \Omega,
$$

where $R_{\text {elong }}$ can be found by considering the areal surface coverage $Z=N \pi R^{2}$ at the elongation transition. In the analysis of droplet growth models, a period of self-similar growth driven by coalescence ensues once nucleation has saturated, characterized by a constant surface coverage of $55 \% .^{2,24}$ This value is also consistent with the concept of a "jamming limit" at $Z=54.7 \%$ found in mathematical models of randomly packed circles on a plane, which should correspond to the initial surface configuration of this selfsimilar growth regime. ${ }^{25,26}$ It is therefore reasonable to assume that in the absence of coalescence, the elongation transition is reached after a slight increase in surface coverage. We find from our KMC simulation data (KMC methodology and data are presented later in the manuscript) that $Z$ converges to $57.7 \%$ at $\theta_{\text {elong }}$ in all cases where coalescence can be neglected. ${ }^{27}$ Thus, a relation

$$
R_{\text {elong }}=\left(0.577 / \pi N_{\text {elong }}\right)^{1 / 2} \approx 3 /\left(7 \sqrt{N_{\text {elong }}}\right)
$$

can be written. Substituting Eq. (9) in Eq. (8) followed by Eq. (8) in Eq. (7)

$$
F_{\text {crit }}=(14 \pi / 9 \Omega) B N_{\text {elong }}^{3 / 2} .
$$

In the initial stages of island nucleation and growth, the competition for adatoms between nucleation and island growth causes the island density to saturate as a very wellestablished function of $F$ and $D$

$$
N_{\text {sat }}=\eta(F / D)^{2 / 7},
$$

where $\eta$ is a pre-factor and the exponent $2 / 7$ is specifically for 3D growth with a critical nucleus size of one atom. ${ }^{1,29-33}$ In the coalescence-free growth regime, the island density remains unchanged past the saturation point and through subsequent growth transitions; thus, we set $N_{\text {elong }}=N_{\text {sat }}{ }^{28}$ Finally, substituting Eq. (11) in Eq. (10) and rearranging for $F_{\text {crit }}$ yields

$$
F_{\text {crit }}=\left(14 \pi \eta^{3 / 2} / 9 \Omega\right)^{7 / 4} B^{7 / 4} / D^{3 / 4} .
$$

Equation (12) constitutes the main result of this study and expresses the relationship between $B, D$, and $F$ that governs the ideal crossover between coalescence-controlled and coalescence-free growth regimes. To offer an explanation of the physical mechanisms described in this relation, we consider the following events during growth: (i) higher diffusivity favors island growth at the expense of nucleation, (ii) this enhanced island growth obstructs coalescence completion for islands that impinge before $N_{\text {sat }}$ is reached, (iii) once $N_{\text {sat }}$ is reached, all subsequent deposition is assumed to feed island growth, which, in combination with large island sizes caused by the earlier growth enhancement, effectively stops coalescence from completing at all. Thus, since a larger portion of the deposited flux contributes to island growth in steps (i) and (ii), less total flux is needed to suppress coalescence and $F_{\text {crit }}$ decreases with increasing $D$. However, if $B$ is increased, the rate of coalescence will be faster for all island sizes. In order to counterbalance this, more material overall 
is required to feed island growth, hence, $F_{\text {crit }}$ increases for increasing $B$. Note however that since both $B$ and $D$ are temperature-dependent (cf. Eqs. (1) and (5)), they will be simultaneously affected if the growth temperature is varied to control growth dynamics. Since the rates of change for $D$ and $B$ in Eq. (12) are not equal ( $B$ grows to the power of $7 / 4$ while $D$ only grows to the power of 3/4) the net effect on $F_{\text {crit }}$ will be dominated by the effects of $B$. This is illustrated by simulation and experimental data presented later in the article.

To test the validity of Eq. (12), we performed KMC simulations seeking to confirm the existence of the two growth regimes by studying the scaling behavior of $\theta_{\text {elong }}$ against $F$, $B$, and $D$. The KMC simulation algorithm is largely based upon previous works. ${ }^{4,34}$ Atoms are deposited at a rate $F$ to random positions on a simple cubic substrate with lattice parameter $a$ and periodic boundary conditions. The simulation box size is $512 \times 512$ lattice sites for all simulations. Adatoms diffuse to nearest-neighboring sites with a diffusivity given by Eq. (5). Once two adatoms meet, they irreversibly form a stable, hemispherical, and immobile island. Impingement between neighboring islands forms a cluster, and a coalescence clock is started for each pair of impinging islands with a total time calculated from Eq. (2). If the coalescence clock reaches the total time, the coalescing islands are removed from the simulation and replaced with a single island centered at their combined center of mass and with their combined size. The elongation transition film thickness $\theta_{\text {elong }}$ is determined as the point when the average cluster size equals two islands. ${ }^{27}$

Two sets of simulations were performed; set 1 was with constant $D=1.14 \times 10^{7} \mathrm{a}^{2} / \mathrm{s}$, over a range of $B=10$ $-4000 \mathrm{a}^{4} / \mathrm{s}$ and $F=0.01-60 \mathrm{ML} / \mathrm{s}$ and set 2 with constant $B=400 \mathrm{a}^{4} / \mathrm{s}$, over a range of $D=489-5.26$ $\times 10^{6} \mathrm{a}^{2} / \mathrm{s}$ and $F=0.02-100 \mathrm{ML} / \mathrm{s}$. The large ranges of $F$ facilitate visualization of the asymptotic behavior of $\theta_{\text {elong, }}$, i.e., when $F \rightarrow 0$ and $\infty$. The variations in $D$ were generated by using a set of $E_{D}$ values for $\mathrm{T}=300 \mathrm{~K}$, though these values are not reported here as the same variations in the parameter $D$ may be generated by varying the temperature. $\theta_{\text {elong }}$ from sets 1 and 2 are visualized as functions of $F$ in Figs. 1(a) and 1(b), respectively. Also, $F_{\text {crit }}$ positions calculated by Eq. (12) (with $\left.\eta=0.25, \Omega=a^{3}\right)^{35}$ for the simulated growth conditions $(B$ and $D)$ are indicated with solid vertical lines. It is seen that $\theta_{\text {elong }}$ exhibits power law dependences on $F$ very close to Eqs. (3) and (4), thus identifying the coalescence-controlled and coalescence-free growth regimes in both figures. Furthermore, it can be seen that the calculated $F_{c r i t}$ values agree very well to the simulated crossover positions. In Fig. 1(a), $F_{\text {crit }}$ shifts nearly two orders of magnitude as $B$ is varied one order of magnitude from $400 \mathrm{a}^{4} / \mathrm{s}$ to $4000 \mathrm{a}^{4} / \mathrm{s}$. To obtain roughly the same shift of $F_{\text {crit }}$ in Fig. 1(b), $D$ must be varied over two orders of magnitude, from $5.26 \times 10^{6} \mathrm{a}^{2} / \mathrm{s}$ to $1.08 \times 10^{4} \mathrm{a}^{2} / \mathrm{s}$, which corroborates the earlier indication of $B$ having a stronger effect on $F_{\text {crit }}$ than $D$.

To complement KMC simulation results, deposition of $\mathrm{Ag}$ vapor on $\mathrm{SiO}_{2}$ was used as a model system to study the scaling behavior of characteristic transition thicknesses at various growth conditions. Owing to its definition,

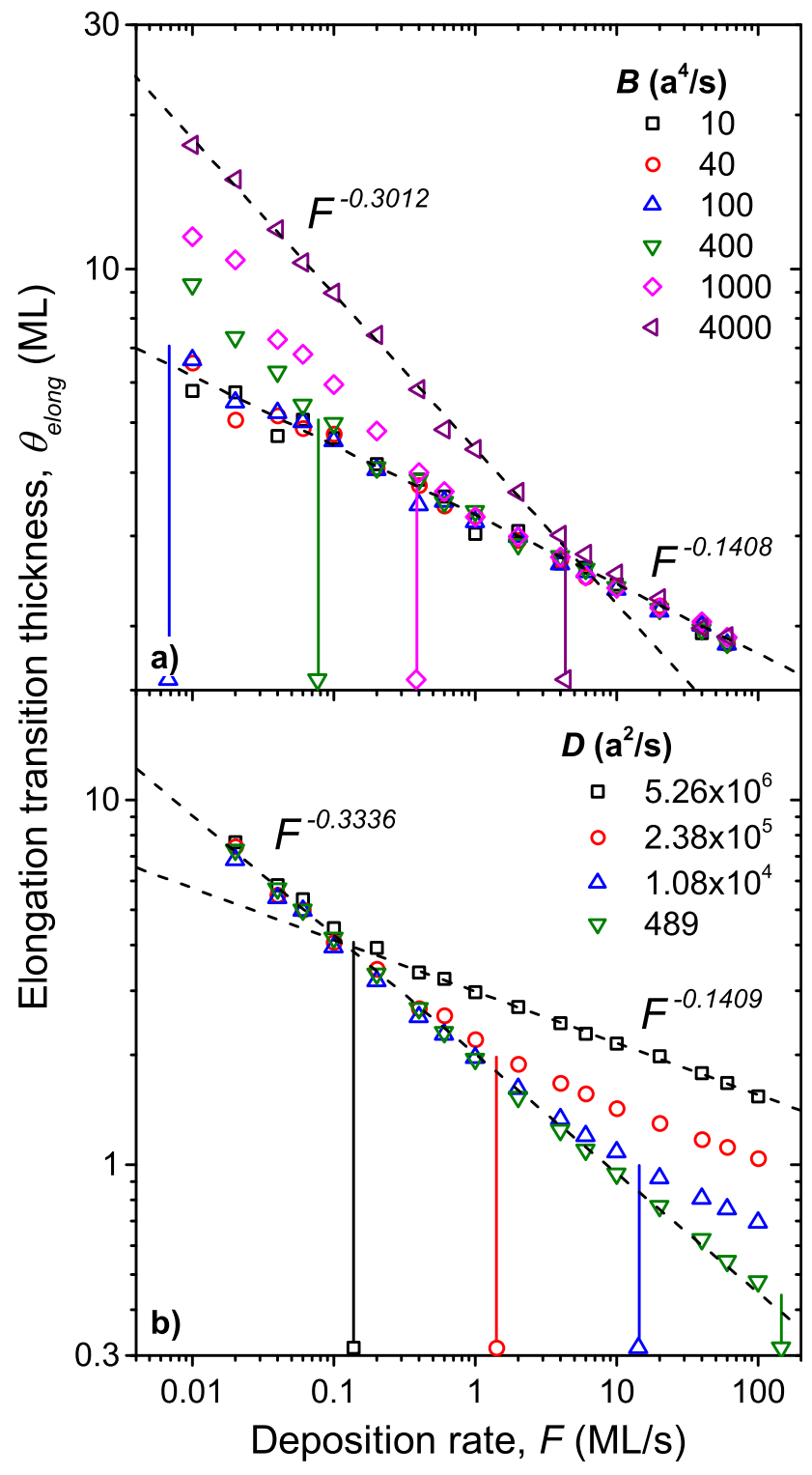

FIG. 1. Elongation transition thickness $\theta_{\text {elong }}$ against deposition rate $F$ from the two simulations sets, (a) set 1 and (b) set 2 . Dashed lines are guides to the eye for identifying the coalescence-free and the coalescence-controlled growth regimes, with exponents -0.14 and -0.33 , respectively. The vertical solid lines indicate crossover positions calculated by Eq. (12) for conditions where the crossover occurs within the range of simulated deposition rates.

experimental measurements at the elongation transition are generally difficult to perform with sufficient accuracy. Therefore, the analogous film thicknesses at percolation $\left(\theta_{\text {perc }}\right)$ and continuous film formation $\left(\theta_{\text {cont }}\right)$ are measured in the real world experiments. Carrey and Maurice suggested, based on KMC simulations, a linear relation between $\theta_{\text {elong }}$ and $\theta_{\text {perc }},{ }^{18}$ and we have recently shown, for deposition of pulsed $\mathrm{Ag}$ vapor on $\mathrm{SiO}_{2}$, that the relation between $\theta_{\text {perc }}$ and $\theta_{\text {cont }}$ appears to be linear as well. ${ }^{36}$ The latter is supported by the idea of hole-filling being the dominant process between percolation and continuous film formation. ${ }^{36,37}$ Based on this, we assume that effects on $\theta_{\text {elong }}$ are reflected in $\theta_{\text {perc }}$ and $\theta_{\text {cont }}$ as well. Continuous $\mathrm{Ag}$ fluxes of various rates $F$, ranging from $5.27 \times 10^{-3}-7.02 \times 10^{-2} \mathrm{ML} / \mathrm{s}$ were generated by sputtering an $\mathrm{Ag}$ target in a vacuum chamber (base pressure $\left.1.3 \times 10^{-6} \mathrm{~Pa}\right){ }^{27}$ Two different growth temperatures were employed, room temperature $(300 \mathrm{~K})$ and $330 \mathrm{~K}$, 


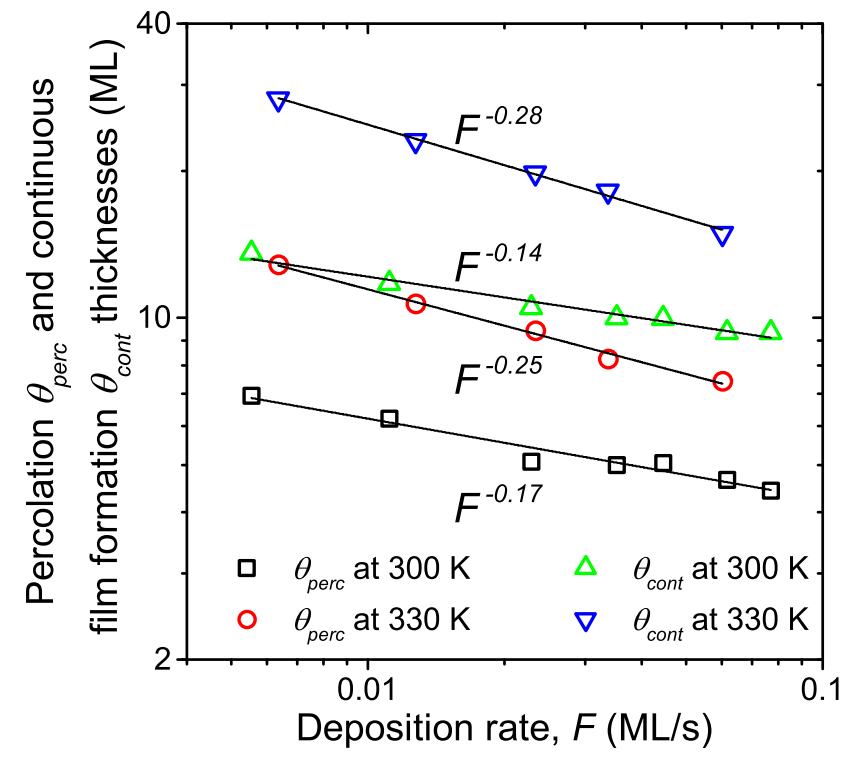

FIG. 2. Measured percolation transition $\theta_{\text {perc }}$ and continuous film formation $\theta_{\text {cont }}$ thicknesses for films deposited at room temperature $(300 \mathrm{~K})$ and elevated temperature $(330 \mathrm{~K})$ as functions of the deposition rate $\boldsymbol{F}$. The data points are fitted with power-law expressions (lines) from which the exponents of $\boldsymbol{F}$ are found.

aiming at changing values of $B$ and $D$. Film growth was monitored in situ using spectroscopic ellipsometry, from which $\theta_{\text {perc }}$ and $\theta_{\text {cont }}$ were calculated by analyzing the ellipsometric data using dispersion models and graphical methods. ${ }^{27}$ Figure 2 shows $\theta_{\text {perc }}$ and $\theta_{\text {cont }}$ obtained in the experiments, and it can be seen that both thicknesses scale linearly with each other $\left(\theta_{\text {cont }} \approx 2 \theta_{\text {perc }}\right)$, in agreement with a previous work of ours. ${ }^{36}$ On the basis of Eqs. (3) and (4) and the simulation results presented in Fig. 1, the scaling exponents in Fig. 2 indicate that deposition at $300 \mathrm{~K}$ resembles the growth behavior in the coalescence-free regime (exponents -0.17 and -0.14 for $\theta_{\text {perc }}$ and $\theta_{\text {cont }}$, respectively), while deposition at $330 \mathrm{~K}$ results in exponents $(-0.25$ to -0.28 for $\theta_{\text {perc }}$ and $\theta_{\text {cont }}$, respectively) that indicate faster island coalescence and thus coalescence-controlled growth. These findings are consistent with Eq. (12) and the KMC data in Fig. 1, which predict a shift of $F_{\text {crit }}$ to larger values and thus coalescence-controlled growth when both $B$ and $D$ are increased for a given vapor deposition rate. It is also seen in Fig. 2 that the scaling exponent in the, presumably, coalescence-controlled regime deviates strongly from the ideal value predicted by Eq. (3) (-0.33). While the possibility of this being a result of the approximate nature of our coalescence description should be acknowledged, it is also conceivable that the experiments at both temperatures occur in or in the vicinity of a wide crossover transition region, similar to those describe for observations made during the growth of $\mathrm{Ag}$ on $\mathrm{SiO}_{2}$ using pulsed vapor fluxes. ${ }^{36}$

In summary, the effects of competition between coalescence and island growth were quantified in an analytical relation that connects three basic quantities governing nanoscale growth morphology: the deposition rate $F$, the substrate diffusivity $D$, and the strength of coalescence $B$. Using this relation, the critical deposition rate, $F_{\text {crit }}$, required to cross between coalescence-controlled and coalescence-free growth regimes can be calculated for given $B$ and $D$ values, and the form of the analytical expression suggested that $F_{\text {crit }}$ is more sensitive to variations of $B$ rather than $D$. Predictions of this relation were compared and validated to kinetic Monte Carlo simulations for growth conditions varying over large ranges of $F, D$, and $B$ values; the comparisons showed very good quantitative agreement. Within the framework of our theory, we interpreted experimental measurement results of $\mathrm{Ag}$ vapor deposition on $\mathrm{SiO}_{2}$ where two growth temperatures $(300 \mathrm{~K}$ and $330 \mathrm{~K})$ were investigated. It was suggested that this material system resembles coalescence-free growth at room temperature $(300 \mathrm{~K})$ while an ongoing transition to the coalescence-controlled growth regime occurs at $330 \mathrm{~K}$.

The ability to predict the occurrence of coalescencecontrolled and coalescence-free growth regimes for different material systems provides insight with respect to the ability to control the rate of coalescence during vapor condensation. This in turn may prove beneficial for tailoring thin film architecture at the nanoscale by using both nucleation and coalescence to access a larger variety of film morphologies and physical attributes. Moreover, by being able to unambiguously determine $F_{c r i t}$ experimentally, the relations derived herein also provide an opportunity to estimate $B$ and $D$ by monitoring the evolution of characteristic transition thicknesses in the different growth regimes as a function of deposition conditions.

The authors would like to acknowledge financial support from Linköping University via the "LiU Research Fellows" program, the Swedish Research Council through contract VR 621-2011-5312 and ÅForsk through the project "Towards Next Generation Energy Saving Windows." Computational resources were provided by the National Supercomputer Center (NSC) at Linköping University. Professor Hans Arwin and Professor Kenneth Järrendahl (Laboratory of Applied Optics, Linköping University) are gratefully acknowledged for providing the in situ spectroscopic ellipsometer.

${ }^{1}$ J. A. Venables, G. D. T. Spiller, and M. Hanbücken, Rep. Prog. Phys. 47, 399 (1984).

${ }^{2}$ P. Meakin, Reports Prog. Phys. 55, 157 (1992).

${ }^{3}$ G. Jeffers, M. A. Dubson, and P. M. Duxbury, J. Appl. Crystallogr. 75, 5016 (1994).

${ }^{4}$ J. Carrey and J.-L. Maurice, Phys. Rev. B 63, 245408 (2001).

${ }^{5}$ X. Yu, P. M. Duxbury, G. Jeffers, and M. A. Dubson, Phys. Rev. B 44, 13163 (1991).

${ }^{6}$ M. Bartelt and J. Evans, Surf. Sci. 298, 421 (1993).

${ }^{7}$ G. S. Bales and D. C. Chrzan, Phys. Rev. B 50, 6057 (1994).

${ }^{8}$ J. G. Amar, F. Family, and P.-M. Lam, Phys. Rev. B 50, 8781 (1994).

${ }^{9}$ F. A. Nichols and W. W. Mullins, J. Appl. Phys. 36, 1826 (1965).

${ }^{10}$ F. A. Nichols, J. Appl. Phys. 37, 2805 (1966).

${ }^{11}$ N. Combe, P. Jensen, and A. Pimpinelli, Phys. Rev. Lett. 85, 110 (2000).

${ }^{12}$ A. D. Brailsford and N. A. Gjostein, J. Appl. Phys. 46, 2390 (1975).

${ }^{13}$ F. Family and P. Meakin, Phys. Rev. A 40, 3836 (1989).

${ }^{14}$ L. Ç. Arslan, C. Sanborn, E. Anzenberg, and K. F. Ludwig, Phys. Rev. Lett. 109, 106102 (2012).

${ }^{15}$ W. W. Mullins and G. S. Rohrer, J. Am. Ceram. Soc. 83, 214 (2000).

${ }^{16}$ S. Grachev, M. De Grazia, E. Barthel, E. Søndergård, and R. Lazzari, J. Phys. D: Appl. Phys. 46, 375305 (2013).

${ }^{17}$ D. McCarthy and S. Brown, Phys. Rev. B 80, 064107 (2009).

${ }^{18}$ J. Carrey and J.-L. Maurice, Phys. Rev. B 65, 205401 (2002).

${ }^{19}$ J. Warrender and M. Aziz, Phys. Rev. B 76, 045414 (2007).

${ }^{20}$ M. Tomellini and M. Fanfoni, J. Phys. Condens. Matter 18, 4219 (2006).

${ }^{21}$ M. Fanfoni, R. Polini, V. Sessa, M. Tomellini, and M. Volpe, Appl. Surf. Sci. 152, 126 (1999). 
${ }^{22}$ P. Jensen, N. Combe, H. Larralde, J. L. Barrat, C. Misbah, and A. Pimpinelli, Eur. Phys. J. B 11, 497 (1999).

${ }^{23}$ By leaving, it is meant that an atom on the island surface relocates such that the hemispherical shape of the island is no longer preserved.

${ }^{24}$ D. Fritter, C. M. Knobler, and D. A. Beysens, Phys. Rev. A 43, 2858 (1991).

${ }^{25}$ D. Stauffer, Phys. Rep. 54, 1 (1979).

${ }^{26}$ E. L. Hinrichsen, J. Feder, and T. Jssang, J. Stat. Phys. 44, 793 (1986).

${ }^{27}$ See supplementary material at http://dx.doi.org/10.1063/1.4900575 for detailed descriptions of experimental methods, mathematical derivations, and additional simulation results.

${ }^{28}$ To avoid confusion regarding the definition of an "island," we use this to signify the primary islands formed in the initial nucleation stage, and imagine these as separate entities even when they are clustered with other islands, until coalescence forms a new, shape-relaxed island. In this way, we may describe an island density also at the elongation transitions.

${ }^{29}$ D. Walton, J. Chem. Phys. 37, 2182 (1962).

${ }^{30}$ Y. A. Kryukov and J. G. Amar, Phys. Rev. B 81, 165435 (2010).

${ }^{31}$ P. Jensen, H. Larralde, M. Meunier, and A. Pimpinelli, Surf. Sci. 412/413, 458 (1998).

${ }^{32}$ H. Brune, G. S. Bales, J. Jacobsen, C. Boragno, and K. Kern, Phys. Rev. B 60, 5991 (1999).

${ }^{33}$ P. T. Stroud, J. Phys. C Solid State Phys. 4, 577 (1971).

${ }^{34}$ P. Jensen, Rev. Mod. Phys. 71, 1695 (1999).

${ }^{35}$ H. Brune, Surf. Sci. Rep. 31, 125 (1998).

${ }^{36}$ V. Elofsson, B. Lü, D. Magnfält, E. P. Münger, and K. Sarakinos, J. Appl. Phys. 116, 044302 (2014).

${ }^{37}$ P. M. Martin, Handbook of Deposition Technologies for Films and Coatings, 3rd ed. (William Andrew Pub., 2009), p. 912. 